\title{
Identifying Innovative National Markets for Technical Consumer Goods
}

\author{
Michael Lynn, Cornell University \\ Betsy D. Gelb, University of Houston
}

Population characteristics that appear to make one nation more or less innovative for technical consumer products are focused on. Three predictors - individualism, uncertainty avoidance and purchasing power - are found to be related to national levels of new product ownership within Europe. The results, focusing on their implications for marketers seeking to export innovative technological consumer goods to Europe and elsewhere, are discussed.

Do some countries have higher adoption rates for innovative technological consumer products than others do? If so, what population characteristics are associated with such national innovativeness? Using data from 16 European nations, this paper explores these questions and then offers implications beyond European boundaries.

The possibility that some nations are more innovative concerning technical consumer goods, and that such innovativeness is associated with predictable population characteristics, can be important for exporters either within or outside the European Community. Of the $\$ 1.3$ trillion in goods imported by the European nations in 1992 (IMF 1993), an estimated 5 per cent could be viewed as "technical" products, everything from household appliances to computers. With the increased popularity of global marketing strategies, it is of course plausible that an exporter will select "Europe" as a market, with little concern for inter-country variations in innovativeness for technological products. However, knowledge concerning the variations in national innovativeness is of practical concern for at least four reasons:

1) An exporter may want just to put a toe in the water and begin export to one or two European nations, and if so, will want to identify more vs. less innovative markets as at least one factor in selecting the nations to target.

2) Even a firm which exports to all of Europe may find its performance differing across countries, and may surmise that these differences are due to sales representation, distributors, or other controllable issues when in fact they are attributable at least in part to the differing innovativeness of various European countries. 
3) Knowledge of predictors of lower levels of innovativeness can lead to informed efforts to counteract the influence of those predictors - e.g. an exporter might allocate more advertising weight to a less innovative country and might change ad copy, or even redesign the product itself, for that country to take into account the reasons for its lack of innovativeness.

4) Once predictors can be identified which are associated with innovativeness for technical consumer products in the case of European nations, they likewise may be helpful for Asia, North America, South America, or Africa in selecting national markets, understanding variations in performance among national markets and/or making marketing mix adjustments to take into account expected variations across nations.

\section{Introduction}

\section{Literature Review}

The concept of innovativeness has a long and rich history in consumer behavior, where innovativeness is the tendency of individual consumers to adopt new products before large numbers of others do (see Gatignon and Robertson, 1985 for a review). However, the idea that nations may also differ in innovativeness is relatively new and has received little research attention. Few studies of crosscountry differences in new product diffusion have been conducted and most of those studies have focused on predicting and explaining national differences in diffusion patterns rather than on assessing the construct of national innovativeness (see for example, Gatignon et al., 1989; Helsen et al., 1993; Takada and Jain, 1991).

One exception to the general neglect of the concept of national innovativeness was provided by Chol Lee (1990). Lee (1990) introduced the concept of national innovativeness, which he defined as "the degree to which a country adopts an innovation relatively earlier than the rest of other countries"( $p$. 40), and he measured this construct with the number of black and white television sets per 1,000 people in a country. Lee (1990) found that this measure of national innovativeness was positively related to a nation's:

Gross Domestic Product per capita;

levels of industrialization and electricity consumption;

form of government (democratic or not);

dominant religion (Protestant or not); 
number of scientists and engineers per 1,000 people;

literacy rate and number of college students per 1,000 people;

number of outgoing tourists per 1,000 people.

\section{Contribution of Current Study}

Lee's (1990) introduction of the concept of national innovativeness and examination of its correlates made a significant contribution to the literature. However, this work can be improved upon in several respects. These potential improvements are identified and discussed below.

Firstly, Lee's (1990) definition of national innovativeness as "the degree to which a country adopts an innovation relatively earlier than the rest of other countries" (p.40), is problematic because it has two incompatible dimensions - the extent of adoption and the earliness of adoption. The extent of adoption can only be defined and measured at a given point in time and the earliness of adoption can only be defined and measured at a given level of adoption, so combining these dimensions creates an indefinite and unmeasurable construct. Of the two dimensions captured by Lee's definition, the extent of adoption seems most relevant to marketers who seek to increase sales of innovations. Thus, we recommend that national innovativeness be defined as "the extent to which a nation's consumers adopt innovative, new products." Given this definition, national innovativeness can be measured by the percapita number of innovative new products owned or by the percentage of individuals or households who own innovative new products. (This use of per capita and percentage indicators means that less innovative, but larger, nations may account for more sales of a new product than do more innovative, but smaller, nations. However, it is important to use per capita and percentage figures rather than simply numbers of products owned and numbers of product owners, in order to differentiate the concept of national innovativeness from the concept of national market size. In making decisions, managers must consider both concepts, but in this paper we focus on national innovativeness and its implications.)

Secondly, Lee's (1990) use of ownership of a single product to measure national innovativeness raises questions about the generalizability of his results. Demographic, economic, geographic and legal factors often affect the adoption of different products differently. For example, mountains block many airwave transmissions, so they might be expected to increase the adoption of cable television, but to decrease the adoption of cellular phones. Lee's use of a single product indicator to measure national innovativeness makes it possible that the predictors of that measure are idiosyncratic. Heightening this concern about the generalizability of Lee's (1990) results are research findings that national differences 
in new product diffusion patterns tend to vary across product categories (Gatignon et al., 1989; Helsen et al., 1993; Takada and Jain, 1991; see also Tansuhaj et al., 1991). Indeed, these findings raise serious questions about the existence of any cross-product consistencies in national innovativeness. To address these questions, research measuring national ownership of several different innovative products is needed.

Thirdly, Lee's (1990) use of demographic, economic and political data as measures of cultural variables raises questions about the interpretation of some of his results. For example, Lee used the presence of a democratic form of government as a measure of national attitudes towards authority and he used the dominance of Protestant religions as a measure of national attitude towards change. Consistent with Lee's interpretations of these measures, research has found that the authoritarianism of national governments is related to national attitudes towards power and that the ratio of Catholics to Protestants is related to national attitudes towards change (Hofstede, 1984). However, these political and religious measures are related to a variety of other cultural values as well. For example, the ratio of Catholics to Protestants is related to attitudes towards power hierarchies, individualism, and traditional sex roles as well as to attitudes towards change (Hofstede, 1984). Thus, it is not clear from Lee's data what cultural values affect a nation's innovativeness for consumer goods. To identify the cultural values underlying national innovativeness, research using more direct measures of the cultural constructs is needed.

Finally, Lee's (1990) inclusion of both industrialized and non-industrialized countries in his study sample limits the usefulness of his research to those marketers seeking to identify the most innovative industrialized nations. Many marketers will be primarily concerned with innovativeness among the industrialized nations, because those nations have wealthier and more accessible markets. Lee's (1990) study does not report separate analyses for industrialized and non-industrialized nations and this omission is significant because relationships that exist within a large, heterogeneous sample may not hold within a smaller, more homogeneous sample. For example, Lee's finding that national innovativeness was highly related to national wealth (per capita GDP) in his sample does not tell us about the effects on national innovativeness of the comparatively small differences in national wealth among industrialized countries. Thus, additional research can be helpful in highlighting the predictors of national innovativeness among the industrialized countries of the world.

In this paper, we build upon Lee's (1990) article. Like Lee's study, our research examines cultural and economic predictors of national innovativeness. However, our paper goes beyond Lee's work and makes a unique contribution by addressing each of the research needs identified above. We use data on 
national ownership of several different innovations to assess the cross-product consistency of national innovativeness and to construct a more generalizable measure of national innovativeness. In addition, we use direct, validated measures of cultural values to more clearly identify some of the values underlying national innovativeness. Finally, we use a sample of European countries in order to better serve those marketers who want to know about the predictors of innovativeness among industrialized nations only.

\section{Predictors and Hypotheses}

The specific predictors of national innovativeness examined in this study are national levels of individualism, uncertainty avoidance and purchasing power. These predictor variables were selected for study because of theoretical relevance and data availability as described below.

Many researchers have pointed out that cultural variables are difficult and expensive to operationalize in international contexts (Helsen et al., 1993; Wind and Douglas, 1972). However, one exceptional source of secondary, international data on cultural values can be found in the work of Hofstede $(1983 ;$ 1984). Hofstede surveyed IBM employees in 50 different countries/regions about workrelated values and found four dimensions underlying the national differences in those values - i.e. power distance, individualism, uncertainty avoidance, and masculinity. Power distance refers to a country's acceptance of status and power differences between people. Individualism refers to a country's tendency toward an "I-consciousness" and a self-orientation as opposed to a "We-consciousness" and a collectivity-orientation. Uncertainty avoidance refers to a country's intolerance of change, risks and uncertainty. Masculinity refers to a country's tendency to value "masculine" goals like money and achievement over "feminine" goals like social relationships and service. Hofstede (1983) developed and published national scores on these four dimensions for 50 different countries/regions.

Although based on work-related survey questions, Hofstede's (1983) indices of power distance, individualism, uncertainty avoidance and masculinity are meaningfully related to a variety of other economic, political and psychological variables (Hofstede, 1984). For example, Lynn, et al. (1993) demonstrated that these value indices were related to cross-country differences in the consumer behaviour of tipping. Milner et al. (1993) argued that these values are likely to be related to crosscountry differences in other forms of consumer behaviour as well. In our opinion, two of these value indices - individualism and uncertainty avoidance - should be related to differences in national innovativeness. 
Individualism could affect national differences in new product diffusion in several different ways. Individualistic cultures value autonomy and individual initiative over group membership and conformity (Hofstede, 1984). This suggests that the more individualistic a country is the more willing its people would be to try a new product before others do (thus, the larger the Bass coefficient for innovation), but the less quickly its people would be to model others' adoption of an innovation (thus, the smaller the Bass coefficient for imitation). Furthermore, individualistic cultures are more hedonistic and materialistic than are collectivistic cultures (Hofstede, 1984; Triandis, 1989). This suggests that the appeal of innovative consumer goods will be greater in more individualistic countries. Assuming that individualism's effects on product appeal dominate its other effects, we hypothesize that new products will be more widely adopted the greater a country's individualism.

Uncertainty avoidance should affect national differences in new product diffusion in a straightforward manner. Purchasing innovative new products involves making changes, taking risks and accepting uncertainty. Uncertainty avoidance reflects a country's tendency to avoid these things (Hofstede, 1984). Therefore, we hypothesize that new products will be less widely adopted the greater a country's uncertainty avoidance (see also Milner et al., 1993). This hypothesis appears to be tautological, but it is not. At a conceptual level, national innovativeness is an economic construct referring to marketplace behaviour while uncertainty avoidance is a cultural construct referring to national values. At an operational level, the two constructs are measured using data collected with different instruments from different samples. Thus, it is neither necessary nor certain that national innovativeness will be related to uncertainty avoidance in this study. The hypothesized relationship between these variables needs to be empirically tested.

The final predictor variable included in this study was national consumer purchasing power. National differences in consumer purchasing power mean that the "average" consumers in some countries are better able to afford innovative consumer products than are the "average" consumers in other countries. Thus, we hypothesize that new products will be more widely adopted in countries with higher levels of consumer purchasing power. Of course, "new" products are often adopted by only a fraction of the population and every industrialized country has a sizeable group of consumers who can afford new consumer goods, so it is possible national innovativeness is independent of the "average" consumer's purchasing power. It is for this reason that we consider the hypothesis worth testing. 


\section{Method}

In order to test the hypotheses it was necessary to obtain measures of national differences in ownership of new products, individualism, uncertainty avoidance and consumer purchasing power. These measures were obtained from secondary sources as described below. Complete data was available for 16 European countries - Austria (AUS), Belgium (BEL), Denmark (DEN), Finland (FIN), France (FRA), West Germany (GER), Great Britain (GRB), Greece (GRE), Ireland (IRE), Italy (ITA), Netherlands

(NET), Norway (NOR), Portugal (POR), Spain (SPA), Sweden (SWE), and Switzerland (SWI).

National innovativeness/new product ownership

A measure of national innovativeness was developed from new product ownership data reported in Reader's Digest Eurodata (RDE). This report summarizes results from a survey of 22,339 consumers in 17 European countries (the 16 listed previously plus Luxembourg) conducted in 1990 for Reader's Digest by Gallup International. The questionnaire was pilot tested in each country, then modified to correct problems of miscomprehension and translated by Gallup field organizations. Each translation was checked by local Reader's Digest editorial personnel, then interviews were conducted with probability samples obtained using the "random route method" in each country. Data on the percentages of households within each country that possessed a cordless telephone, a telephone answering machine, a home computer, a microwave, a compact disc player, a video camera and a satellite dish were used as indicators of national innovativeness. These particular products included in the index were selected because they were relatively new, and possessed by fewer than 30 per cent of European households.

\section{Individualism and Uncertainty Avoidance}

Measures of national individualism and uncertainty avoidance were obtained from the work of Hofstede $(1983 ; 1984)$. Hofstede developed national index scores on these variables from responses to questionnaires on work-related values that were administered to 116,000 employees of IBM in 50 different countries. The questionnaires were written in English and translated by local IBM employees into their native languages. Translations were then checked by some of the company's bilingual managers. Hofstede's $(1983 ; 1984)$ national samples were not representative of their respective populations. However, they were well matched on demographic and occupational variables. This means that the absolute values of Hofstede's cultural indices may be systematically biased, but that the bias should be approximately the same for every country. Therefore, the data should validly capture cross- 
country differences in the relevant values. Indeed, Hofstede (1984) has amassed a great deal of evidence attesting to the validity of these indices.

\section{Purchasing Power Parity}

A measure of national levels of consumer purchasing power was obtained from the Book of Vital World Statistics (The Economist, 1990). This book contains United Nations estimates of purchasing power parity (PPP), which adjusted countries' 1988 Gross Domestic Products for costs of living. These PPP measures are the best available means of comparing consumer purchasing power across countries (see Kravis et al., 1986; Summers and Heston, 1991; Walker, 1995; Ward, 1985).

\section{Results}

\section{Consistency of Indicators}

We selected seven indicators of national innovativeness for inclusion in this study - the percentage of households in a nation owning a cordless telephone, a telephone answering machine, a home computer, a microwave, a compact disc player, a video camera and a satellite dish. These indicators had very different means and variances, which gives undue weight to those indicators with the largest variances, so they were standardized before statistical analysis. A principal components analysis of these standardized indicators produced two components/factors with eigen values greater than 1.0. All of the indicators except ownership of satellite dishes loaded more than 0.50 on the first factor and only ownership of satellite dishes loaded more than 0.50 on the second factor (see Table I). Therefore, we interpreted the first factor as national innovativeness and developed an index of this construct by averaging all of the standardized indicators except ownership of satellite dishes. This index of national innovativeness (or new product ownership) had a coefficient alpha of 0.86 and was used in the analyses reported below.

\section{Predictors of National Innovativeness}

The index of national innovativeness was significantly correlated with national individualism ( $\mathrm{r}=$ $0.77, p<0.05)$, uncertainty avoidance $(r=-0.58, p<0.05)$, and purchasing power $(r=0.80, p<0.05)$. Analyses of the separate indicators produced similar, but not identical, patterns of results (see Table II). Particularly notable is the fact that purchasing power did not significantly predict ownership of cordless telephones. This relationship may have been weakened by the impact of geographic, technological and legislative factors that have affected national differences in the adoption of this particular product. 
Another possibility is that national purchasing power failed to significantly predict ownership of cordless telephones because cordless telephones are relatively inexpensive. This later possibility suggests that product expensiveness may moderate the relationship between national innovativeness and national purchasing power.

The national levels of uncertainty avoidance and individualism were moderately correlated with purchasing power ( $r$ 's $=-0.53$ and 0.67 ) and with one another $(r=-0.68)$. This multicolinearity among predictor variables raises the possibility that some of the predictors' relationships with the measure of national innovativeness were simply due to the confounding effects of the other predictors. This possibility was addressed by conducting a simultaneous multiple regression analysis that assessed the relationship of each predictor with the index of national innovativeness after statistically controlling for the potentially confounding effects of the other predictors. The statistical power of this multivariate analysis is low because of our small sample of only 16 nations. Nevertheless, two of the three predictors remained statistically significant. National innovativeness remained significantly related to individualism (partial $r=0.46, t(12)=1.81$, one-tailed $p<0.05)$ and to purchasing power (partial $r=0.53, t(12)=2.63$, one-tailed $p<0.02$ ), but not to uncertainty avoidance (partial $r=-0.03, t(13)=-0.09$, n.s.). Thus, the relationships of individualism and of purchasing power with national innovativeness cannot be attributed to the confounding effects of the other predictors. The negative relationship between uncertainty avoidance and national innovativeness may be attributable to the other predictors, but additional research with a larger sample (and more statistical power) should be conducted before such an attribution is made.

\section{Discussion}

This study found that cross-product consistencies in national innovativeness do exist and that national innovativeness is related to national levels of individualism, uncertainty avoidance and purchasing power. These results have several implications for basic theory and research in marketing as well as for marketing practice.

Theoretical implications

The results of this study make several contributions to basic theory and research. They have implications for:

the generalizability of the "national innovativeness" concept;

the theoretical explanations for differences in national innovativeness;

the usefulness of Hofstede's value indices in consumer research. 
Each of these implications is discussed below.

Generalizability of national innovativeness. National levels of new product ownership have many product specific causes. For example, the adoption of a new product is affected by the taxes and regulations imposed on its acquisition. National differences in tax and regulatory policies are not constant across different products, so they represent non-generalizable causes of national levels of new product ownership. It is possible that product-specific factors like taxes and regulations are so powerful that there are little or no cross-product consistencies in national levels of new product ownership. Such a lack of generalizability would severely compromise the utility of the national innovativeness concept. Therefore, we tested the cross-product consistency of national levels of new product ownership via factor analysis. In that analysis, a common factor accounted for $\mathbf{4 0}$ to 70 per cent of the variance in national ownership levels for six out of seven new products. This result means that national levels of new product ownership have some generalizability and that the national innovativeness concept has some utility. Of course, the products used in this study were all technical consumer goods, so we interpret our results as reflecting national innovativeness for these types of goods only.

Explanation for national innovativeness. There are theoretical reasons (outlined in the introduction) for believing that national differences in individualism, uncertainty avoidance and purchasing power have a causal effect on national innovativeness. Consistent with these theoretical considerations, we found that all three of the hypothesized independent variables were in fact related to national innovativeness. The relationships observed in this study were only correlational, so firm conclusions about causality are not possible. However, the consistency of our observations with the hypotheses fails to disconfirm the theorized causal processes and thereby adds to our confidence in these causal explanations.

Utility of Hofstede's indices. Hofstede's value indices have been empirically related to a variety of economic and social variables (see Hofstede, 1984). Recently, Milner et al. (1993) argued that these indices may also shed light on national differences in consumer behaviour. However, there are at least two reasons to question this argument. Firstly, Hofstede's indices measure work-related values and it is possible that national cultures have different value priorities for people's roles as workers than for their roles as consumers. Secondly, Hofstede collected his data in the late 1960s and early 1970s and it is possible that differences in national values have changed in the intervening years. Our data sheds light on the validity of these concerns. Specifically, the fact that two of Hofstede's indices were related to national innovativeness in this study suggests that the values measured by these indices are applicable to national differences in consumer behaviour and that any changes in national values since the 
measurement of the indices have not been large enough to completely undermine the indices' predictive validities. Thus, our study provides some empirical support for Milner et al's belief that Hofstede's indices are potentially useful tools for international consumer research.

\section{Practical Implications}

As noted at the outset of this paper, the results of our study have practical implications for international marketers. Specifically, these results have implications for selecting national markets; evaluating national marketing efforts; controlling the marketing mix; identifying other innovative national markets.

Of course, innovativeness is not the only, or even the most important factor to consider when engaging in these activities, but it is one factor that should be considered. Accordingly, the managerial implications of this factor are discussed below, with the understanding that management decisions should also be based on a consideration of market size and other factors that are not discussed.

Selecting national markets. The first implication applies to marketers who wish to begin export of a technical consumer innovation just to one or two European nations, either because of resource limitations or because they want to test the acceptability of the product in Europe before increasing their investment. For these managers, as Table III shows, the measure of national innovativeness developed for this study from product-specific RDE ownership data points to Great Britain, the Netherlands, Norway, Finland, Sweden, Switzerland, Belgium and France as the most innovative European nations. Assuming that the "toe-in-the-water" approach may be motivated by a wish to assess the acceptability of an innovative offering in Europe, it should of course be pointed out that beginning with the presumably most receptive countries offers an advantage but also a disadvantage. If the product fares poorly, one or more of several explanations can be valid, but the one which at least can be disparaged is the notion that "this culture just resists innovation." Conversely, of course, success for the innovative product cannot lead a marketer who first targets one or two "most susceptible" countries to expect comparable success throughout Europe.

Evaluating marketing efforts. The second implication of these data concerns firms exporting technical consumer goods to several or all European countries, but experiencing different degrees of success in different markets. Managers in such a firm can look at where they are most vs. least successful, and whether the variations in success roughly match variations in national innovativeness. If 
this is so, managers may at least tentatively attribute performance differences across countries to differences in innovativeness rather than attributing them to salespersons, distributors, or the like.

Controlling the marketing mix. A third implication is that it may be prudent to handle a number of "marketing mix" variables differently in less innovative countries. Products themselves could be made to more closely resemble earlier products from which they are derived, or other products currently on the market. For example, in a country where an automatic clothes dryer is an innovation but a washing machine is not, the dryer could be made to resemble a washer. Distribution could involve familiar channels, rather than emphasizing the "newness" of a product by placing it in a new kind of store. Even pricing could be a consideration if a marketer is entering the European market only in one country; a less innovative national market suggests that the conventional "skimming" approach to pricing an innovation might need to be employed for a shorter period of time if not abandoned altogether.

Of course, advertising offers a number of possibilities for increasing sales in less innovative countries. One possibility is to budget extra funds in less innovative countries, funds which can be employed with some sophistication based on knowledge of the indicators on which the countries score behind the European "innovators." High uncertainty avoidance, a characteristic associated with Belgium and France, suggests an emphasis on warranties and promises to replace products found unsatisfactory. Relatively low individualism scores, as in Norway, suggest the value of an "everybody has one" approach to promotion. Furthermore, it is possible in any non-innovative country to emphasize in advertising copy that an innovation is a "better version of what you have," rather than something entirely new.

Identifying other innovative national markets. Finally, the results of this study make it possible to look beyond Europe for innovative national markets, using the indicators which predicted innovation within Europe. A wish to examine data outside of Europe might be expressed, for example, by a manager who had taken to heart the message that the less industrialized countries are "where the global action is" (Saporito, 1993). As Table III shows, however, industrialized countries show up as the most promising non-European nations on the innovation-receptive dimensions - the USA, Canada, Australia, and New Zealand rank highest. Likewise, industrialized countries show up best within geographic sub-groups, such as Asia. By contrast, the picture is universally disappointing in Africa and South America. One might expect our use of purchasing power rather than Gross Domestic Product or household income to be the economic measure to help in providing a favorable profile for developing nations, but none shows up as ranking high. Likewise, neither of the two other variables suggests high levels of national innovativeness anywhere in Africa or South America. Of course, finding a country with unattractive scores across the board does not preclude it as a market for a technical innovation. 
However, it does suggest looking elsewhere first if innovativeness for technical consumer goods is a construct of consequence to a marketer.

\section{References}

The Economist (1990, Book of Vital World Statistics, Random House, New York, NY.

Gatignon, H., Eliashberg, J. and Robertson, T.S. (1989, "Modelling multinational diffusion patterns: an efficient methodology", Marketing Science, Vol. 8, Summer, pp. 231-47.

Gatignon, H. and Robertson, T.S. (1985, "A propositional inventory for new diffusion research", Journal of Consumer Research, Vol. 11, March, pp. 849-67.

Helsen, K., Jedidi, K. and DeSarbo, W.S. (1993, "A new approach to country segmentation utilizing multinational diffusion patterns", Journal of Marketing, Vol. 57, October, pp. 60-71.

Hofstede, G. (1983, "National cultures in four dimensions", International Studies of Management and Organization, Vol. 13, January-February, pp. 46-74.

Hofstede, G. (1984, Cultures Consequences: International Differences in Work-related Values, Abridged Edition, Sage Publications, London.

International Monetary Fund (1993, Direction of Trade Statistics Yearbook, International Monetary Fund, Washington, DC.

Kravis, I.B., Heston, A. and Summers, R. (1986, Phase IV: World Comparisons of Purchasing Power and Real Product for 1980, United Nations, New York, NY.

Lee, C. (1990, "Determinants of national innovativeness and international market segmentation", International Marketing Review, Vol. 7, pp. 39-49.

Lynn, M., Zinkhan, G. and Harris, J. (1993, "Consumer tipping: a cross country study", Journal of Consumer Research, Vol. 20, December, pp. 478-87.

Milner, L.M., Fodness, D. and Speece, M.W. (1993, "Hofstede's research on cross-cultural work-related values: implications for consumer behaviour", in Van Raaj, W.F. and Bamossy, G.J. (Eds), European Advances in Consumer Research, Vol. 1, Association for Consumer Research, Provo, UT, pp. 70-6.

Reader's Digest (1990, Reader's Digest Eurodata, Reader's Digest Association, London.

Saporito, B. (1993, "Where the global action is", Fortune, Vol. 128, special issue Autumn/Winter, pp. 635.

Summers, R. and Heston, A. (1991, "The Penn World Table (Mark 5): an expanded set of international comparisons, 1950-1988", The Quarterly Journal of Economics, May, pp. 327-68. 
Takada, H. and Jain, D. (1991, "Cross-national analysis of diffusion of consumer durable goods in Pacific Rim countries", Journal of Marketing, Vol. 55, April, pp. 48-54.

Tansuhaj, P., Gentry, J.W., John, J., Manzer, L.L. and Cho, B.J. (1991, "A cross-national examination of innovation resistance", International Marketing Review, Vol. 8 No. 3, pp. 7-20.

Triandis, H.C. (1989, "The self and social behaviour in differing cultural contexts", Psychological Review, Vol. 96, pp. 506-20.

Walker, C. (1995, "The global middle class", American Demographics, September, pp. 40-6.

Ward, M. (1985, Purchasing Power Parities and Real Expenditures in the OECD, OECD, Paris.

Wind, Y. and Douglas, S.P. (1972, "International marketing segmentation," European Journal of Marketing, Vol. 6, pp. 17-25. 